\title{
Nanomosaic Network for the Detection of Proteins Without Direct Electrical Contact
}

\author{
Jean Gamby, ${ }^{*}$ Jean-Pierre Abid, Bernard Tribollet, and Hubert H. Girault
}

\begin{abstract}
$\boldsymbol{A}$ nanomosaic network of metallic nanoparticles for the detection of ultralow concentrations of proteins is reported, which uses two planar microelectrodes embedded in a microchip that permit generation of capacitive coupling to the nanomosaic system without the need for direct electrical contact with the channel. By tailoring the microchannel surface using a sandwich configuration of polyethylene terephthalate/gold nanoparticles/poly(L-lysine), the surface charge can be modified following biomolecular interactions and monitored using a noncontact admittance technique. This nanodevice system behaves like a tunable capacitor and can be employed for the detection of any kind of molecule. The femtomolar detection of an anionic protein, such as $\beta$-lactoglobulin in phosphatebuffered saline medium, is taken as an example.
\end{abstract}

Keywords:
- admittance
- capacitors
- nanoparticles
- polymers
- proteins

\section{Introduction}

In the last decade, the emergence of nanoengineering has opened up new ways of developing highly sensitive sensors in the areas of life science, catalysis, environment, food, and medicine. Up to now, optical techniques have been preferred due to their sensitivity and nondestructive character. Among them, linear and nonlinear optical techniques, such as confocal fluorescence microscopy, ${ }^{[1-3]}$ surface plasmon resonance (SPR) imaging, ${ }^{[4-6]}$ hyper-Rayleigh scattering (HRS) ${ }^{[7-9]}$ surface second-harmonic generation $(\mathrm{SHG}),{ }^{[10-12]}$ and sumfrequency generation, ${ }^{[13-16]}$ were widely developed for detecting ultralow concentrations of biomolecules or studying monolayers of molecules at interfaces. In traditional fluorescence techniques, the detection is achieved through the ability to tag or attach fluorescent dye to the target molecules, and a limit of detection of $100 \mathrm{pg} \mathrm{mL}^{-1}$ can be easily reached.

[*] Dr. J. Gamby, Dr. B. Tribollet

Laboratoire des Interfaces et Systèmes Electrochimiques UPR15 du CNRS and Université Pierre et Marie Curie Paris VI 4 Place Jussieu, case postale 133, 75252 Paris (France)

Fax: $(+33)$ 1-44-27-40-74

E-mail: gamby@ccr.jussieu.fr

Dr. J. Gamby, Dr. J.-P. Abid, Prof. H. H. Girault Laboratoire d'Electrochimie Physique et Analytique Ecole Polytechnique Fédérale de Lausanne Institut des Sciences et Ingénierie Chimiques 1015 Ecublens (Switzerland)
Recently, several nonlinear optical approaches have been investigated for achieving single-molecule detection ${ }^{[2,3,11]}$ based on the centrosymmetry principle. In SHG and HRS, the signal is proportional to the number density of molecules and the hyperpolarizability per molecule. At interfaces between two centrosymmetric systems, the centrosymmetry is broken and monolayers of molecules can be easily detected. In the past decade, the SPR technique has been demonstrated to be a powerful technique for sensing low concentrations of biomolecules, and monitoring the adsorption of target biomolecules on specifically prepared glass slides containing a thin gold film. ${ }^{[17-20]}$ The principle is based on monitoring the changes in the optical reflectivity of the gold substrate modified with biomolecules. The sensitivity is due to the efficiency of the collective excitation of the conduction electrons near the gold surface.

Sophisticated techniques, such as the Love-mode surface acoustic wave, have been described for studying the quantity of adsorbed biomolecules at the solid/liquid interface. ${ }^{[21]}$ In comparison to the SPR optical technique, a slight modification is made on the quartz substrate, which is patterned with a double-finger interdigitated electrode for launching a Lovemode acoustic wave at a frequency of $123.5 \mathrm{MHz}$. During the process of adsorption, the modification of the phase shift can be converted to a frequency shift and thus related to the bound mass of biomolecules. Another alternative is the use of a quartz crystal microbalance which is a gravimetric sensor. ${ }^{[22-24]}$ The adsorption of biomolecules or molecules onto 
the surface of the quartz modifies the resonance frequency of the quartz crystal. This variation can be directly related to the variation of mass by using the Sauerbrey relation, ${ }^{[25]}$ and thus allows the estimation of the quantity of biomolecules adsorbed on the sensor surface. This technique allows the detection of $87.5 \mathrm{ng} \mathrm{mL}^{-1}$ of biomolecules.

More recently, the possibility of enhancing the electrical response of devices using a network of nanomaterials has opened new pathways for sensing ultralow concentrations of molecules or biomolecules. In such systems, assembling metallic or semiconducting nanomaterials on insulators provides information on the electrical structure at the metal/solution interface through measurement of the capacitance. Based on the same concept, electrochemical impedance spectroscopy provides a thorough method of probing biomolecular interactions at conductive and semiconductive surfaces, with related applications in immunosensors, DNA sensors, and enzyme biosensors. ${ }^{[26,27]}$ With this method, the bias of the sensing electrode potential can be controlled, and the frequency of the alternating potential difference imposed is in the kilohertz range. Contactless techniques, with electrodes that are not located in direct contact with the measured solution, have recently been introduced to probe ultralow concentrations of ions or molecules. The special configuration of the surface electrodes avoids any contact with the probed solution, and thus problems such as electrical coupling, bubble generation, electrode passivation, or contamination can be avoided.

Two configurations were considered: contactless conductimetric detection and supercapacitive admittance tomoscopy. The former technique was previously developed in chip electrophoresis for detecting ions in low-concentration conditions, ${ }^{[28-31]}$ in which there is a minimal stray capacitance between the two microelectrodes (a purely resistive case). The second configuration was developed for studying the highfrequency main capacitive current contribution through coupled microelectrodes. The configuration allows the detection of biomolecules in a region of low concentration in the nano to picomolar range, and opens new possibilities for achieving online detection of low concentrations of molecules and ions. ${ }^{[32]}$ Herein, we present a new biodevice designed for the enhancement of the sensitivity of detection using a network of metallic nanoparticles. The mosaic configuration allows a limit of detection in the femtomolar range to be reached. The device is based on a sandwich configuration of nanomaterials and polymers: polyethylene terephthalate/gold nanoparticles/poly(L-lysine) (PET/NPs/PLL). The surface charge is modified during adsorption of the macromolecules and monitored using capacitive admittance tomoscopy. This nanodevice system behaves like a tunable capacitor and can be employed to detect any kind of molecule.

\section{Results and Discussion}

\subsection{Admittance Measurement Approach}

The principle of the detection is based on applying an ac voltage with constant amplitude and measuring the current through the device (see Experimental Section). Typically, an ac voltage perturbation $\left(V_{1}\right)$ with frequency ranging from $1000 \mathrm{~Hz}$ to $1 \mathrm{MHz}$ and a fixed amplitude is applied through the microelectrodes, and the output signal $\left(V_{2}\right)$ is detected using an electrochemical interface (Solartron, FRA 1255B). The output current is converted into a voltage with a current converter (OPA 602) and amplified using a low-noise preamplifier (Stanford Research Systems, SR560; Figure 1a). Then, the output signal $\left(V_{2}\right)$ is recorded and can be connected to the total admittance of the probed system.

As detailed in the Experimental Section, the microchip device consists of a PET microchannel involving two planar microelectrodes separated by a gap of $100 \mu \mathrm{m}$ and located $5 \mu \mathrm{m}$ under the microchannel (see Figure $2 \mathrm{a}-\mathrm{d}$ ). In that configuration, the physical response of the device can be regarded as a combination of capacitors and resistor for modeling the different contributions arising from the different interfaces. Considering the detection scheme in Figure 1c, it is clear that a capacitive coupling $\left(C_{0}\right.$ corresponds to the parallel capacitance and $C$ is the capacitance of the electrical interface) is taking place at high frequency in the dielectric system of the

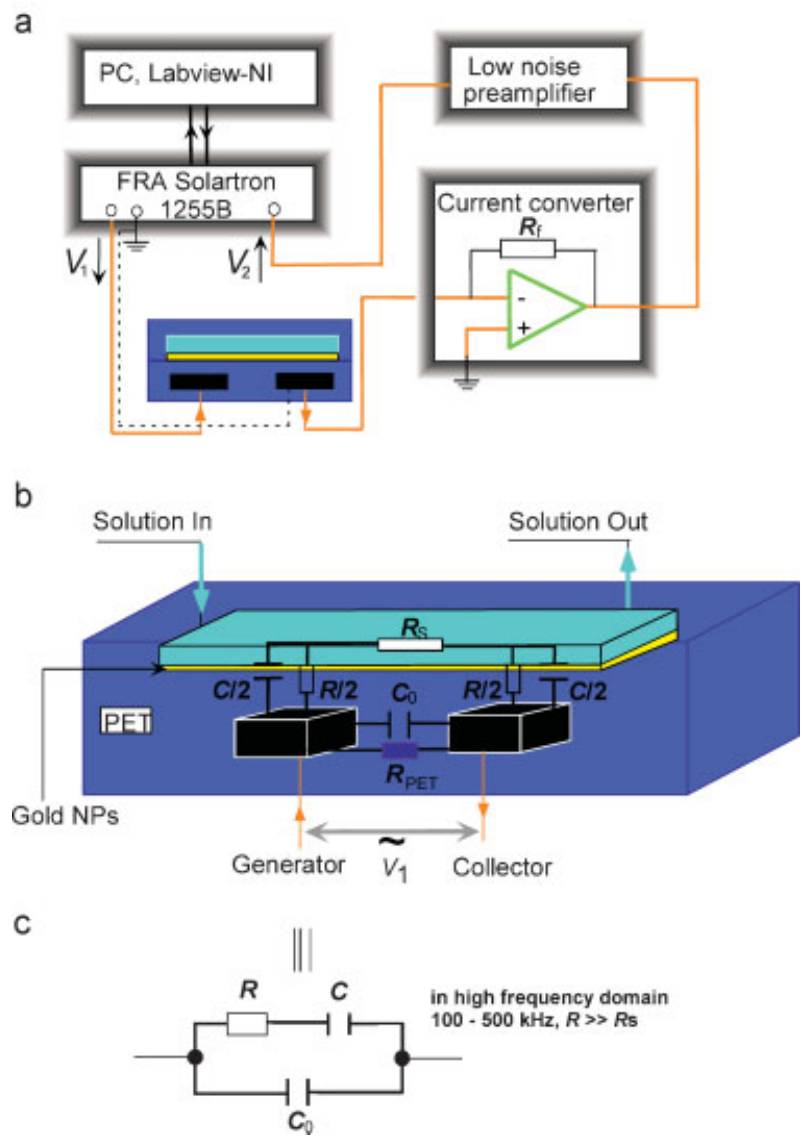

Figure 1. a) Detection of capacitive admittance in the noncontact mode. $V_{1}$ represents the input signal, $V_{2}$ the output signal, and $R_{\mathrm{f}}$ the feedback resistor on the pickup amplifier. b) Schematic view of the microelectrodes/PET/microchannel interface; $R, R_{\mathrm{PET}}$, and $R_{\mathrm{S}}$ denote the resistance of the interface, of the bulk PET between the electrodes, and of the solution, respectively. $C$ and $C_{0}$ are the capacitance of the interface and the stray capacitance between the two microelectrodes, respectively. c) Equivalent circuit of the biodevice. 

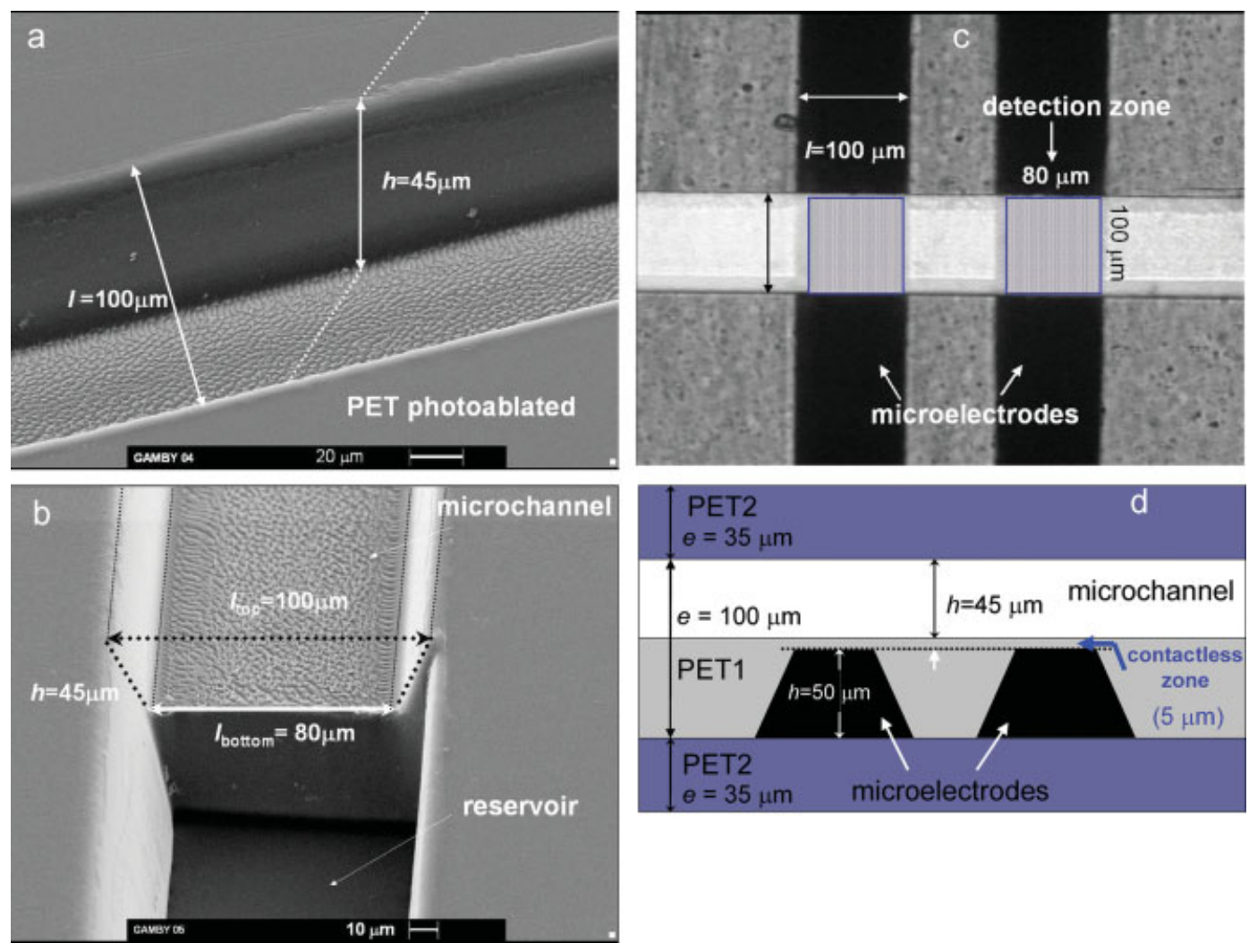

Figure 2. Scanning electron microscopy (SEM) images of a) the PET photoablated microchannel with a cross section of $45 \times 100 \mu \mathrm{m}$ and a length of $1.4 \mathrm{~cm}$; b) trapezoidal section of the microchannel; and c) the planar microelectrodes. Vertically, the two band electrodes beneath the horizontal flow channel. The detection zone represents the area where capacitive coupling takes place. d) Side view of the contactless zone, which is about $5 \mu \mathrm{m}$ and represents the thickness separation between the flow microchannel and the planar microelectrodes.

electrode/PET/channel interface, which arises from direct coupling between the microelectrodes. The parallel capacitance $C_{0}$ originates from the all-stray capacitance between the microelectrodes $C_{0, \text { electrodes }}$ and the stray capacitance to the supporting microstructure, $C_{0, \mathrm{PET}}$. In the high-frequency regime, the solution resistance $R_{\mathrm{S}}$ may be disregarded since the resistance of the interface becomes predominant. Hence, increasing the concentration of analyte induces the increase of the admittance. By considering the product information from melinex (surface resistivity and volume resistivity at $23^{\circ} \mathrm{C}$ are equal to $10^{-13} \Omega \mathrm{cm}^{-2}$ and $10^{-15} \Omega \mathrm{cm}$, respectively) and using Equation (1), the theoretical bulk insulated polymer resistance, $R_{\mathrm{PET}}=7.8 \times 10^{14} \Omega$, has been evaluated from the relation between the resistivity of the thin PET film, the surface area of the detector $S\left(8 \times 10^{-9} \mathrm{~m}^{2}\right)$, and the film thickness $l\left(5 \times 10^{-6} \mathrm{~m}\right)$, as shown in Figure 2a-d.

$R_{\mathrm{PET}}=\frac{\rho l}{S}$

As a consequence, $R_{\mathrm{PET}}$ between the microelectrodes and the microchannel through the bulk of the PET substrate is large enough and can be disregarded. ${ }^{[32]}$

Using noncontact microelectrodes, that is, microelectrodes isolated from the measured media by PET, the measured noncontact capacitance $C_{0}$ can be expressed as the combina- tion of $C_{0, \text { electrodes }}$ and $C_{0, \mathrm{PET}}$ [Eq. (2)]:

$1 / C_{0}=1 / C_{0, \mathrm{PET}}+1 / C_{0, \text { electrodes }}$

where $C_{0 \text {,electrodes }}$ is the interfacial capacitance in the case of insulating layer absence and is dependent on the species adsorbed or attached onto the surface of the channel. $C_{0, \mathrm{PET}}$ is the capacitor formed through the dielectric material and is related to $\varepsilon^{*}$, the complex permittivity of the polymer [Eq. (3)]:

$C_{0, \mathrm{PET}}=\frac{\varepsilon_{0} \varepsilon * S}{l}$

where $\varepsilon_{0}$ is the vacuum permittivity, $S$ is the effective area covered by the capacitor, and $l$ is the thickness of polymer from the microelectrodes to the microchannel. $\varepsilon^{*}=\varepsilon_{\mathrm{r}}-j \varepsilon_{\mathrm{s}}$, where $\varepsilon_{\mathrm{r}}$ is the relative permittivity associated with the polarization and $\varepsilon_{\mathrm{s}}$ the dielectric loss associated with the conductance. In the case of PET, the relative permittivity results from both $\alpha$-relaxation correlated to the semicrystalline microstructure and to $\beta$-relaxation induced by local motion of $\mathrm{C}=\mathrm{O}$ polar side groups.

In the present configuration, the theoretical PET stray capacitance, $C_{0, \mathrm{PET}}=8.5 \times 10^{-14} \mathrm{~F}$, was evaluated from the relation between the permittivity of the thin PET film and its 
dimension, given by [Eq. (4)]:

$C_{0, \mathrm{PET}}=\frac{\varepsilon_{0} \varepsilon_{r} S}{l}$

where $\varepsilon_{0}\left(8.854 \times 10^{-12} \mathrm{~F} \mathrm{~m}^{-1}\right)$ is the vacuum permittivity, $\varepsilon_{\mathrm{r}}$ (3.0) is the relative permittivity of the thin PET film, $S\left(8 \times 10^{-9} \mathrm{~m}^{2}\right)$ is the surface area of the detector, and $l\left(5 \times 10^{-6} \mathrm{~m}\right)$ is the film thickness (see Figure $\left.2 \mathrm{c}, \mathrm{d}\right)$.

The output voltage is given by the gain equation, which accounts for the frequency dependence of the cell admittance. The gain $G$ can be estimated using the following expressions [Eqs. (5-7)]:

$G=-\frac{V_{2}}{V_{1}}=-k \times R_{\mathrm{f}} \times Y(\omega)$

with

$Y(\omega)=\frac{j \omega\left(C_{0}+C\right)-\omega^{2} R C C_{0}}{1+j \omega R C}$

or

$Y(\omega)=\frac{j \omega\left(C_{0}+C\right)\left[1+j \omega\left(\frac{C C_{0}}{C_{0}+C}\right)\right]}{1+j \omega R C}$

where $V_{1}$ and $V_{2}$ are the input and output voltages in volts, respectively, $j$ is the imaginary unit, $\omega$ the angular frequency in $\operatorname{rad~s}^{-1}, Y$ the total admittance of the cell, $R$ the resistance of the interface in $\Omega, R_{\mathrm{f}}$ the feedback resistance, $k$ the preamplifier factor, $C$ the capacitance of the interface, and $C_{0}$ the all-stray capacitance in farads.

Preliminary experiments with a voltage of $0.5 \mathrm{~V}$ and frequency ranging from $1000 \mathrm{~Hz}$ to $1 \mathrm{MHz}$ were performed for understanding and modeling the processes involved in this detection approach. Admittance measurements were performed on the device filled with a buffer solution (called the test buffer), and the modulus of the admittance was obtained (see Figure 3). Using the buffer, an increase of the admittance modulus $\left|V_{2} / V_{1}\right|$ with increasing frequency is observed until the cutoff frequency is reached at $2915.3910^{3} \mathrm{rad} \mathrm{s}^{-1}$ or $464 \mathrm{kHz}$.

The evolution of the admittance modulus establishes clearly that the cell can be regarded as a capacitive system, where the capacitive coupling takes place at frequencies higher than $10^{5} \mathrm{rad} \mathrm{s}^{-1}$. The mutual use of the OPA 602 current converter and the filter limit the spectral range to a cutoff frequency of $\omega_{\mathrm{C}}=10^{6.46} \mathrm{rad} \mathrm{s}^{-1}$ (Figure 3, inset). The feedback resistance and the low-noise preamplifier gain are fixed to give $k R_{\mathrm{f}}=54 \times 10^{3} \Omega$. To further explain the variation of the admittance amplitude with respect to the frequency, the results are modeled using the equivalent circuit displayed in Figure $1 \mathrm{~b}$.

The best fit for the data of Figure 3 according to the fixed parameter $R_{\mathrm{f}} k=54 \times 10^{3} \Omega$ yields a cell resistance $R=2 \times 10^{8} \Omega$, an interfacial capacitance $C=7 \times 10^{-13} \mathrm{~F}$ (channel filled), and an all-stray capacitance $C_{0}=1.2 \times 10^{-11}$ F. Due to the fact that the noncontact capacitance $C_{0}$ is found to be higher than the capacitance of the electrical interface $C$,

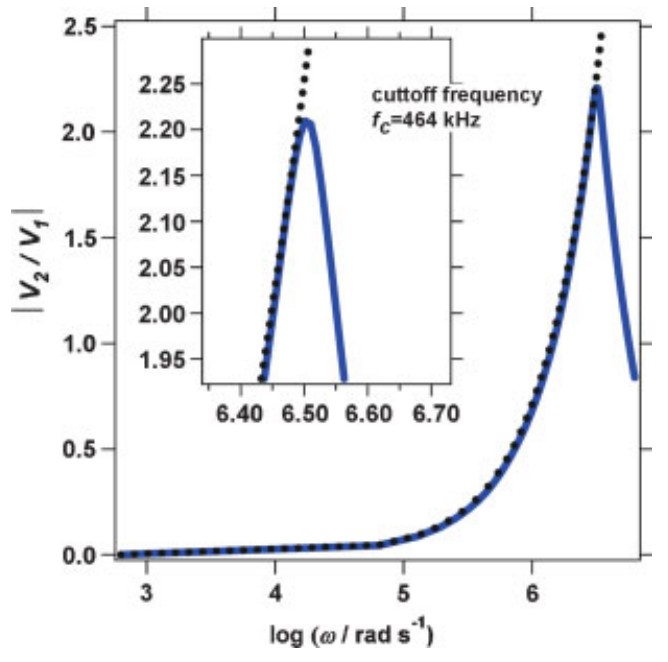

Figure 3. Admittance modulus of a phosphate-buffered saline (PBS) solution $(\mathrm{pH} 7)$ in the microchannel depending on the angular frequency $\left(10^{2.8}\right.$ to $\left.10^{6.8} \mathrm{rad} \mathrm{s}^{-1}\right)$. The solid line represents the experimental results and the dotted line the simulated curve with $k R_{\mathrm{f}}=54 \times 10^{3} \Omega$, $R=2 \times 10^{8} \Omega, C_{0}=1.2 \times 10^{-11} \mathrm{~F}$, and $C=7 \times 10^{-13} \mathrm{~F}$ using the configuration electrode/PET/channel.

the major process involved in the detection scheme is the capacitive coupling. Numerical simulations were investigated to quantify and qualify the capacitive coupling zone for the admittance amplitude response; $|Y|$ is represented in terms of $\log \left|V_{2} / V_{1}\right|$. Figure 4 shows Bode plots with different numerical values of $C_{0}$.

Two regimes can be considered: at lower stray capacitance $\left(C_{0}<C=1.2 \times 10^{-13} \mathrm{~F}\right)$ and at higher levels of $C_{0}\left(C_{0}>C\right)$. In

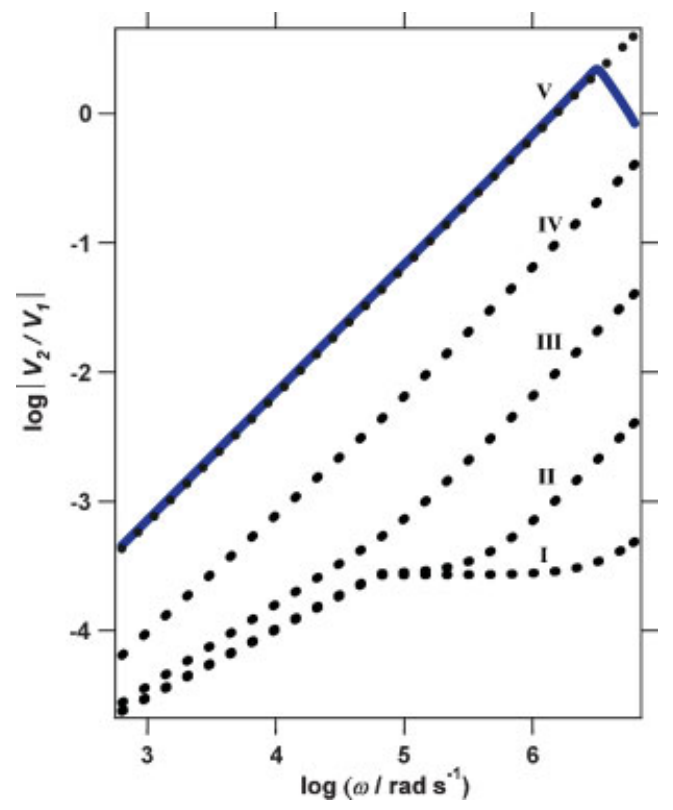

Figure 4. Simulation in Bode-plot representation of the admittance amplitude response of the detector cell with respect to the stray capacitance when a capacitive coupling $\left(C\right.$ and $C_{0}$ ) takes place in the dielectric system of the electrode/PET/channel. Dotted lines from bottom to top: $1.2 \times 10^{-15}, 1.2 \times 10^{-14}, 1.2 \times 10^{-13}, 1.2 \times 10^{-12}$, and $1.2 \times 10^{-11} \mathrm{~F}$ with the same $k \times R_{\mathrm{f}}=54 \times 10^{3} \Omega, R=2 \times 10^{8} \Omega$, and $C=7 \times 10^{-13} \mathrm{~F}$ ); solid line: experimental results. 
Table 1. Dependence of the admittance modulus on $C_{0}$.

\begin{tabular}{lcc}
$\begin{array}{l}\text { Bode } \\
\text { plot }\end{array}$ & Admittance modulus \\
\hline I & $|Y|=\omega \times C$ & $|Y|=1 / R$ \\
II & $|Y|=\omega \times C$ & $\left.|Y|=1 / R \times\left[\left(C_{0}+C\right) / C\right)\right]|Y|=\omega \times\left(C+C_{0}\right)$ \\
III-V & $|Y|=\omega \times C$ & $|Y|=\omega \times\left(C+C_{0}\right)$ \\
\hline
\end{tabular}

the low stray capacitance regime (see Bode plots I and II in Figure 4), a linear increase of the admittance modulus is observed with increasing angular frequency, whereas at high angular frequency a plateau region is observed. In this case, Equation (7) can be simplified and corresponds to a single-pole high-pass filter with resistance $R$ and capacitance $C$. At higher angular frequency a new linear contribution is observed (see Table 1 and Bode plots II-V) due to different levels of stray capacitance. Simultaneously, one can note that the plateau is attenuated (plots II, III) until its total disappearance when the capacitive coupling between $C$ and $C_{0}$ increases. In the present configuration (Bode plot $\mathrm{V}$ ), the evolution of the admittance amplitude with respect to angular frequency shows clearly the importance of the contribution of the stray capacitance on the measurement of the capacitance, where the capacitive coupling takes place at frequencies higher than $10^{5} \mathrm{rad} \mathrm{s}^{-1}$.

Any modification of the microelectrode/PET/microchannel interface leads to an increase of the capacitive coupling $\left(C+C_{0}\right)$. At a fixed angular frequency (the cutoff frequency $\left.\omega_{\mathrm{C}}\right)$, any chemical modification of the microchannel wall induces an increase of capacitive coupling, and thus induces the modification of the admittance modulus.

To better improve the efficiency of the device, the strategy of inserting charging species on insulators has been envisaged. On the conductor side, microelectrodes are made using graphite ink loaded with gold NPs, whereas on the filled microchannel side the specific surface area is increased by adsorbing gold NPs onto the walls of the flow channel. It must be pointed out that the chemical modification of the surface implies the variation of the density of charge, the dielectric constant, and thus of the admittance. In this nanomosaic configuration, any adsorption of molecules on gold NPs will induce the modification of the capacitance of the electrode/ $\mathrm{PET} / \mathrm{microchannel} \mathrm{interfaces.}$

\subsection{Gold Nanoparticle Adsorption in Microchannels}

Configuration involving nanomosaic modification and bare PET surfaces was envisaged and a higher sensitivity was reached for the nanomosaic device. Briefly, the modification of the microchannel is achieved by the adsorption of gold NPs onto the surface of the channel to form a connected system of gold NPs and thus enhance the surface charge of the PET surface. The gold NPs were synthesized through the reduction of an auric chloride salt with trisodium citrate. ${ }^{[33]}$ To ensure a positive charge around the NPs, the colloidal solution is treated with cetyltrimethylammonium chloride, as reported by Weiser. ${ }^{[34]}$ The modification of the channel is performed in four steps. First, the channel is filled with PBS (10 mM), which is taken as a reference for all the experiments. In step 2,

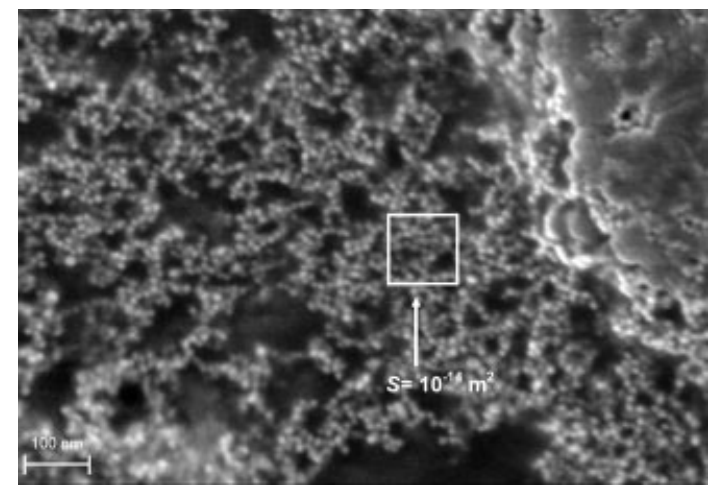

Figure 5. SEM-field-emission gun image of the PET microchannel after surface modification with gold NPs.

modification of the PET channel is obtained by filling the microchannel with a solution of gold NPs and drying it overnight. To remove unattached NPs, the microchannel is rinsed with ethanol and water. In step 3, growth of gold NPs was performed by using hydroxylamine hydrochloride as a growing agent and gold salt in the microchannel. In the last step, the gold NPs are post-functionalized using thiols (3-mercaptopropanoic acid). After each step, the channel is filled with a buffer to point out an increase of interfacial charges (see the Experimental Section for further details).

The image in Figure 5 shows the surface of the channel upholstered with gold NPs. The NPs have an average size of $19 \mathrm{~nm}$ and a standard deviation of $2 \mathrm{~nm}$. The particles were electrostatically stabilized with citrate ions, to yield a surface effective charge of 234 e $\left(3.79 \times 10^{-16} \mathrm{C}\right)$ and an average particle density of $5 \times 10^{15}$ particles $\mathrm{m}^{2}$.

The admittance amplitude experiments are performed with an input voltage fixed at $500 \mathrm{mV}$. To study the effect of surface modification, admittance measurements are performed on bare and nanomosaic modified microchannels. The admittance modulus of the nanomosaic device is recorded and compared to the results obtained for the bare device. Due to the response range of the sensor, all the experiments were performed at a frequency ranging from $10^{2.8}$ to $10^{6.46} \mathrm{rad} \mathrm{s}^{-1}$.

The modification of the PET surface with gold NPs induces an increase of the device admittance amplitude at a cutoff angular frequency of $10^{6.46} \mathrm{rad} \mathrm{s}^{-1}(464 \mathrm{kHz})$. Each step (Figure 6,1-4) of the modification leads to the enhancement of the device admittance modulus. As displayed in Figure 6, the data show a net increase of the admittance modulus when the gold NPs are adsorbed (step 2), reaching a maximum amplitude at the cutoff angular frequency. As discussed previously, the admittance can be regarded as a direct measurement of the surface charge of the PET/solution interface and modeled using a capacitor with a variable capacitance, which is dependent on the species adsorbed onto the surface of the PET microchannel. The optimization of the device configuration is crucial to obtain an ultralow detection level of molecules. The optimal enhancement is achieved for a device made with the $\mathrm{PET} / \mathrm{NPs} /$ growth/mercaptopropionic acid (step 4) configuration. This latter is employed for detecting femtomolar concentrations of proteins, such as $\beta$-lactoglobulin B from bovine milk. 


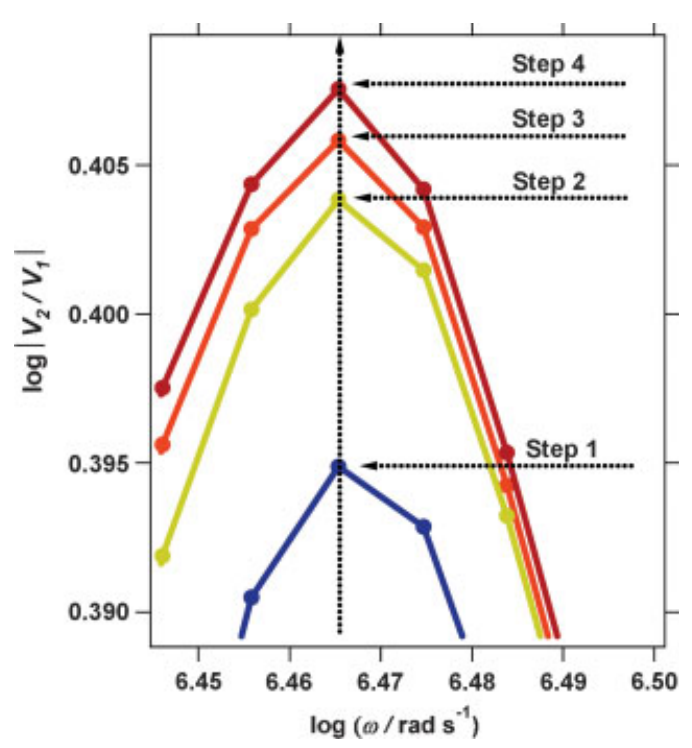

Figure 6. Evolution of the gain at $\omega_{\mathrm{C}}$ for bare and modified devices using a buffer solution. From bottom to top: step 1, bare channel; step 2, adsorption of gold NPs; step 3: growth procedure using hydroxylamine hydrochloride; step 4: post-functionalization using thiols (3-mercaptopropanoic acid).

\subsection{Femtomolar Detection of $\beta$-Lactoglobulin on Sensor Surface}

As reported previously by Bowden ${ }^{[35]}$ the direct adsorption of macromolecules or biomolecules onto the surface of gold provokes the denaturation of the proteins. To avoid any chemical modification, gold NPs were modified using thiols (3-mercaptopropanoic acid). An additional functionalization of the gold NPs/thiols was made using PLL to increase the number of charged sites. In the field of gold electrode modification and characterization, many authors suggest a roughly parallel orientation of PLL on carboxylic acid/alkyl thiol. ${ }^{[35]}$ Also, several investigations of functionalized surfaces have shown some basic protein immobilized by adsorption on carboxylic acid-terminated self-assembled monolayers of the alkyl thiol/gold type. ${ }^{[35-38]}$ In our case, schematic multilayers of a cationic polymer film of PLL were assembled for increasing the possible admittance of the system, and thus allowing detection by immobilization of an anionic protein, such as $\beta$-lactoglobulin. The sandwich system is described in Figure 7.

Each microchannel is modified through steps 1 to 4 , and the procedure is achieved by adsorption of PLL as portrayed in Scheme 1. Admittance measurements were performed for concentrations of $\beta$-lactoglobulin ranging from $4.525 \times 10^{-16}$ to $4.525 \times 10^{-6} \mathrm{M}$. The evolution of the admittance amplitude with respect to the concentration of $\beta$-lactoglobulin is displayed in Figure 8a-d. Increasing the concentration of $\beta$-lactoglobulin B results in an increase of the admittance amplitude until a plateau is reached with a maximal concentration value higher than $4.525 \times 10^{-10} \mathrm{M}$ (see Figure $8 \mathrm{~b}$ and $\mathrm{d}$ ). The admittance measurements show an excellent reproducibility with a negligible variation of the signal. In the field of biosensors, these results emphasize the possibility of detecting subfemtomolar concentrations of protein. Due to

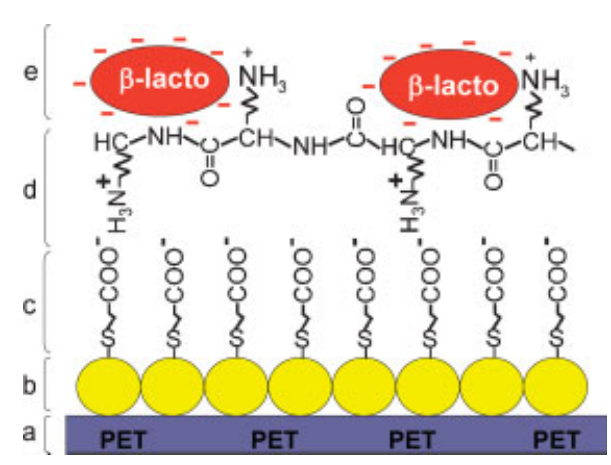

Figure 7. PET surface coating for $\beta$-lactoglobulin detection. a) Bare PET microchannel (step 1); b) gold NPs and growth procedure using hydroxylamine (steps 2 and 3); c) post-functionalization using 3-mercaptopropanoic acid (step 4); d) adsorption of PLL; e) detection of $\beta$-lactoglobulin.

the fact that the response is purely inherent to the interface charge, the device is not limited to the detection of one type of macromolecule.

\section{Conclusions}

An ultrasensitive nanomosaic device based on admittance modulation is designed and employed for detecting traces of biomolecules without direct electrical contact. In this noncontact mode, the system is comparable to a dielectric capacitor and allows capacitive coupling to be observed in the kilohertz frequency region of the electrical current. In the case of the detection of bio-lactoglobulins, a limit of detection of $4.525 \times 10^{-16} \mathrm{M}$ has been easily reached. The experimental results show a very good reproducibility with negligible variation of the signal. The principle is based on the possibility to tune the surface charge and modify the dielectric constant of the medium. The main process governing the electrical properties of the sensor is the adsorption of charged protein at a highly sensitive PET/gold NP/PLL sandwich network. In fact, at $\mathrm{pH} 7$ the $\beta$-lactoglobulin $(\mathrm{pI}=5.1)^{[39]}$ is negatively charged and its adsorption on the surface PLL layer causes a change of the charged region in the Gouy-Chapman layer. This kind of device is not exclusively dedicated to a particular class of molecules and can be employed for online detection in microfluidic applications.

\section{Experimental Section}

Chemicals: Tetrachloroauric acid $\left(99.999 \%, \mathrm{HAuCl}_{4} \cdot 3 \mathrm{H}_{2} \mathrm{O}\right)$ was purchased from Aldrich. Trisodium citrate $\left(95 \%, \mathrm{C}_{6} \mathrm{H}_{5} \mathrm{O}_{7} \mathrm{~N}\right.$ $\mathrm{a}_{3} \cdot 2 \mathrm{H}_{2} \mathrm{O}$ ) was obtained from Fluka. Hydroxylamine hydrochloride was purchased from Alfa Aesar. The glassware and magnetic stirring beads were always cleaned prior to use with freshly prepared a.u. regia $\left(1: 3 \mathrm{HNO}_{3} / \mathrm{HCl}\right)$ followed by rinsing with ultrapure water (Millipore, conductivity $0.8 \mu \mathrm{S} \mathrm{cm}$ ). Mercaptopropanoic acid (98\%), PLL, $\boldsymbol{\beta}$-lactoglobulin B from bovine milk, and PBS tablets were purchased from Sigma-Aldrich. The $\mathrm{pH}$ of the PBS solution $(10 \mathrm{~mm}$ ) was fixed at 7 with solutions of $\mathrm{NaCl}$ and $\mathrm{HCl}$. 

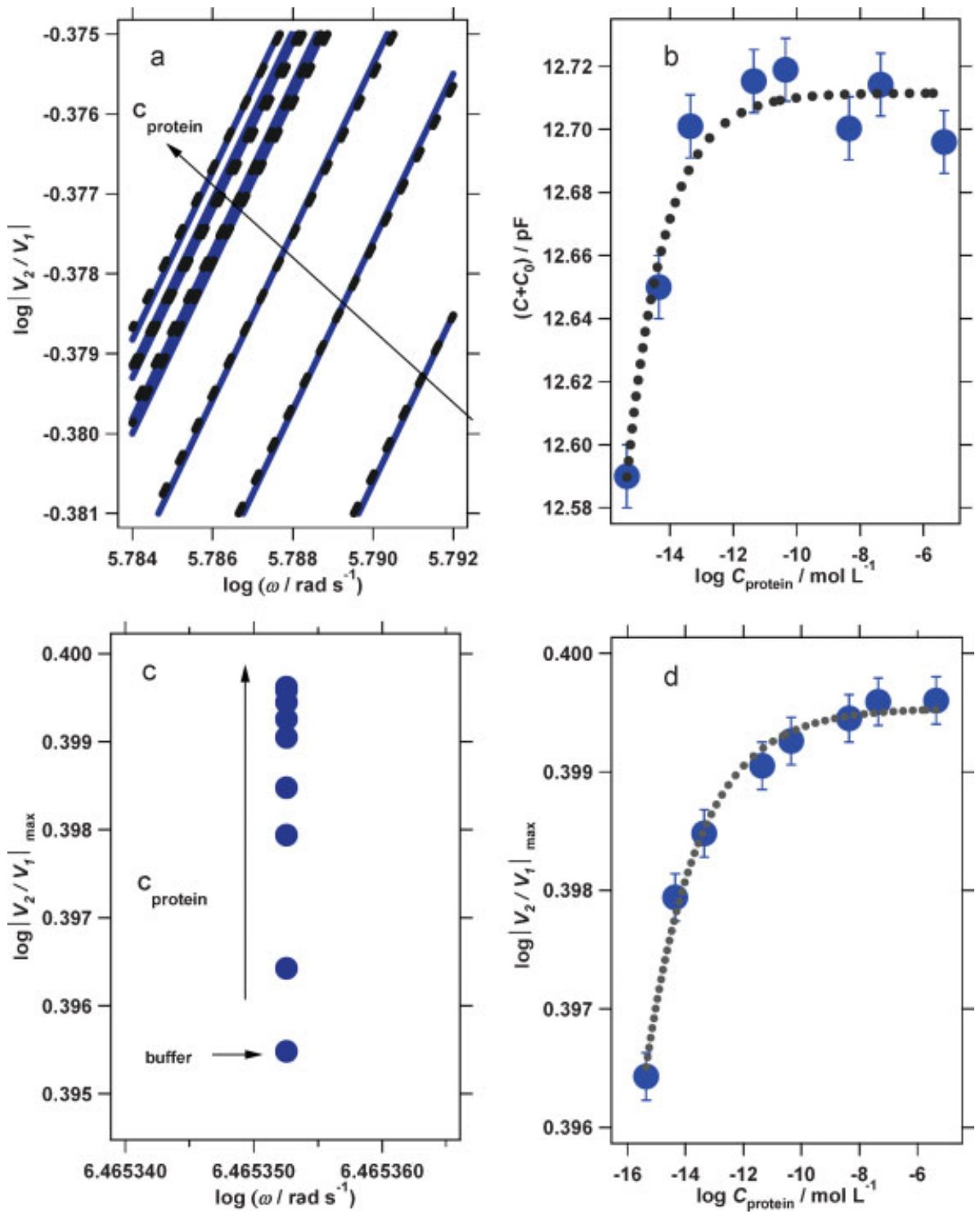

Figure 8. Evolution of the admittance with respect to the concentration of $\beta$-lactoglobulin. a) Experimental angular frequency response of the admittance (solid line) and theoretical fit using Equation (2) (dotted line) with different bulk concentrations of $\beta$-lactoglobulin. Solid line: from bottom to top: $C_{\text {protein }}=4.425 \times 10^{-16}, 4.425 \times 10^{-15}, 4.425 \times 10^{-14}, 4.425 \times 10^{-12}$, $4.425 \times 10^{-11}, 4.425 \times 10^{-9}, 4.425 \times 10^{-8}$, and $4.425 \times 10^{-6} \mathrm{M}$ ); dotted line: best simulation of $\left(C+C_{0}\right)$ capacitance in the case of the adsorption of $\beta$-lactoglobulin onto the nanomosaic surface. Dotted line from bottom to top: $\left(C+C_{0}\right)=12.590 \times 10^{-12}, 12.650 \times 10^{-12}, 12.70 \times 10^{-12}$, $12.715 \times 10^{-12}, 12.718 \times 10^{-12}, 12.70 \times 10^{-12}, 12.714 \times 10^{-12}$, and $12.696 \times 10^{-12} \mathrm{~F}$. b) Evolution of the capacitive coupling with respect to the bulk concentration. c) Vertical evolution of the admittance amplitude at cutoff frequency with respect to the bulk concentration. d) Experimental evolution of the admittance with respect to the bulk concentration.

Synthesis and characterization of gold NPs: The procedure, often referred to as the Turkevich method, derives its name from the work of Turkevich and Stevenson ${ }^{[34]}$ and uses trisodium citrate as the nucleating and reducing agent. A solution of tetrachloroauric acid $(95 \mathrm{~mL})$ with a gold content of $5 \mathrm{mg}$ by weight was heated to boiling point under vigorous stirring. Then, a $1 \%$ sodium citrate aqueous solution ( $5 \mathrm{~mL}$ ) was added. The solution was stirred and kept at boiling conditions for another $45 \mathrm{~min}$. After the introduction of the citrate solution, a purple color appeared which then turned to ruby red. Before the color changes were observed, there was an induction or waiting period of $5 \mathrm{~min}$, which was due to the formation of acetone dicarboxylic acid as precursor, a product of the oxidation of citrate. Acetone dicarboxylic acid plays the roles of precursor, reducing, and nucleating agent. The first stage is the oxidation of citrate into acetone dicarboxylic acid followed by the reduction of gold ions into atoms. The acetone dicarboxylic acid molecule has two groups per unit, which may form a bond with the gold ion. For gold NPs, the organizational role of acetone dicarboxylic acid is the key step for the control of the size and shape of the particles. In the case of gold NPs prepared by reduction with citrate, nucleus buildup is achieved through complexes formed between both $\left[\mathrm{AuCl}_{2}\right]^{-}$and acetone dicarboxylic acid. After the formation of a significant number of nuclei, particle growth occurs through deposition and reduction of gold ions onto the surface of the particles, to yield well-dispersed NPs. The position of the resonance is located at $2.38 \mathrm{eV}(520 \mathrm{~nm})$ and is characteristic of gold NPs with an average size of $20 \mathrm{~nm}$.

Hydroxylamine method in microchannel: Hydroxylamine hydrochloride in aqueous solution is well known to be a growing agent. ${ }^{[33]}$ This method takes advantage of the fact that hydroxylamine does not act as a nucleation agent. When the growth medium is introduced with nuclei, the gold salt is reduced by the hydroxylamine and the metallic gold so formed is deposited on the surface of the nuclei so that they increase in size but not in number. The growth of gold NPs was then developed by adding constant volumes of hydroxylamine hydrochloride ( $1 \mu \mathrm{L}, 2.7 \%$ by weight) and gold salt $(1 \mu \mathrm{L}, 1 \%)$ to the microchannel. In the absence of acetone dicarboxylic acid, no further nucleation occurred, as hydroxylamine hydrochloride does not support nucleation and acts only as a reducing agent.

Microelectrodes and microchip fabrication: PET sheet was photoablated $^{[40]}$ using a UV-excimer laser (Argon Fluor Excimer at 193 nm; Lambda Physik LPX 2051, Göttingen, Germany) to obtain a trapezoidal microchannel with a depth of $45 \mu \mathrm{m}$, a width of $100 \mu \mathrm{m}$, and a length of $1400 \mu \mathrm{m}$ (see Figure 2a,b). After photoablation, the debris produced, such as CO and oxides, was removed by cleaning with isopropanol. The channel structure was then sealed with a PET/polyethylene (PET/PE) layer with a 
thickness of $35 \mu \mathrm{m}$ (Morane, Oxon, UK) at $135^{\circ} \mathrm{C}$ and a pressure of 2 bar (see PET2 in Figure 2d). On the other side of the film, two parallel microchannels with a depth of $50 \mu \mathrm{m}$ and a width of $100 \mu \mathrm{m}$ were photoablated perpendicularly to it, as portrayed in Figure $2 c$. The distance separation between these two parallel microchannels was $100 \mu \mathrm{m}$. The two channels were then filled with a mixture of commercial graphite ink and gold NPs (Electra $\Omega$ ED5000 series obtained from Electra Polymers). After curing at $60^{\circ} \mathrm{C}$ for $4 \mathrm{~h}$, the device consisted of a PET membrane with a microchannel sandwiched between two PET films (PET2 in Figure 2d).

\section{Acknowledgements}

The authors would like to thank Valerie Devaud (LEPA-EPFL) for her technical help, Françoise Pillier (CNRS-LISE) and Stephan Borensztajn (CNRS-LISE) for accessibility to microscopy, and the European Science Foundation for financial support through action "COST D33".

[1] L. Li, S. Sosnowski, C. E. Chaffey, S. T. Balke, M. A. Winnik, Langmuir 1994, 10, 2495-2497.

[2] M. A. Osborne, W. S. Furey, D. Klenerman, S. Balasubramanian, Anal. Chem. 2000, 72, 3678-3881.

[3] I. Sokolov, Y. Y. Kievsky, J. M. Kaszpurenko, Small 2007, 3, 419-423.

[4] a) C. E. Jordan, A. G. Frutos, A. J. Thiel, R. M. Corn, Anal. Chem. 1997, 69, 4939-4947; b) E. A. Smith, W. D. Thomas, L. L. Kiessling, R. M. Corn, J. Am. Chem. Soc. 2003, 125, 6140-6148; c) A. W. Wark, H. J. Lee, R. M. Corn, Anal. Chem. 2005, 77, 3904-3907.

[5] G. Steiner, V. Stablinskas, A. Hubner, C. Khune, R. Salzer, J. Mol. Struct. 1999, 509, 265-273.

[6] M. Kim, K. Park, E.-J. Jeong, Y.-B. Shin, B. H. Chung, Anal. Biochem. 2006, 351, 298-304.

[7] Y. C. Shen, Z. M. Tang, M. Z. Gui, J. Q. Cheng, X. Wang, Z. H. Lu, Chem. Lett. 2000, 10, 1140.

[8] S. Ghosh, A. Krishnan, P. K. Das, S. Ramakrishnan, J. Am. Chem. Soc. 2003, 125, 1602-1606.

[9] P. C. Ray, Angew. Chem. 2006, 118, 1169-1172; Angew. Chem. Int. Ed. 2006, 45, 1151-1154.

[10] R. Antoine, P. F. Brevet, H. H. Girault, D. Bethell, D. J. Schiffrin, Chem. Commun. 1997, 19, 1901-1902.

[11] A. Alparone, S. Millefiori, Chem. Phys. Lett. 2005, 416, 282-288.

[12] S. Cattaneo, K. Miettinen, E. Vuorimaa, H. Lemmetyinen, M. Kauranen, Chem. Phys. Lett. 2006, 419, 492-495.

[13] G. A. Somorjai, Appl. Surf. Sci. 1997, 121, 1-19.

[14] G. Kim, M. Gurau, J. Kim, P. S. Cremer, Langmuir 2002, 18, 2807-2811.

[15] J. Wang, S. M. Buck, M. A. Even, Z. Chen, J. Am. Chem. Soc. 2002, 124, 13302-13305.
[16] M. C. Henry, Y. Yang, R. L. Pizzolatto, M. C. Messmer, Langmuir 2003, 19, 2592-2598.

[17] W. M. Mullett, E. P. C. Lai, J. M. Yeung, Methods 2000, 22, 77-91.

[18] J. Homola, Anal. Bioanal. Chem. 2003, 377, 528-539.

[19] G. J. Wegner, A. W. Wark, H. J. Lee, E. Codner, T. Saeki, S. Fang, R. M. Corn, Anal. Chem. 2004, 76, 5677-5684.

[20] M. H. Zareie, X. Xu, M. Cortie, Small 2007, 3, 139-145.

[21] C. Zhou, J. M. Friedt, A. Angelova, K. H. Choi, W. Laureyn, F. Frederix, L. A. Francis, A. Campitelli, Y. Engelborghs, G. Borghs, Langmuir 2004, 20, 5870-5878.

[22] a) J. J. Garcia-Jareno, C. Gabrielli, H. Perrot, Electrochem. Commun. 2000, 2, 195-200; b) S. Al Sana, C. Gabrielli, H. Perrot, J. Electrochem. Soc. 2003, 150, 444-449.

[23] F. Hoeoek, B. Kasemo, T. Nylande, C. Fant, K. Scott, H. Elwing, Anal. Chem. 2001, 73, 5796-5804.

[24] C. Tamerler, M. Duman, E. E. Oren, M. Gungormus, X. Xiong, T. Kacar, B. A. Parviz, M. Sarikaya, Small 2006, 2, 1372-1378.

[25] G. Sauerbrey, Z. Phys. 1959, 155, 206-222.

[26] S. De Luca, M. Florescu, M. E. Ghica, A. Lupu, G. Palleschi, C. M. A. Brett, D. Compagnone, Talanta 2005, 68, 171-178.

[27] H. Peng, C. Soeller, J. Travas-Sejdic, Macromolecules 2007, 40, 909-914.

[28] a) J. Tanyanyiwa, E. M. Abad-Villar, P. C. Hausser, Electrophoresis 2004, 25, 903-908; b) P. Kuban, P. C. Hauser, Electrophoresis 2004, 25, 3387-3397.

[29] E. Baltussen, R. M. Guijt, G. Van der Steen, F. Laugere, S. Baltussen, G. W. K. Van Dedem, Electrophoresis 2002, 23, 2888-2893.

[30] a) A. J. Zemann, Trends Anal. Chem. 2001, 20, nos. 6 and 7; b) K. Mayrhofer, A. J. Zemann, E. Schnell, G. K. Bonn, Anal. Chem. 1999, 71, 3828-3833; c) A. J. Zemann, E. Schnell, D. Volgger, G. K. Bonn, Anal. Chem. 1998, 70, 536-567.

[31] J. A. Fracassi da Silva, N. Guzman, C. L. Do Lago, J. Chromatogr. A 2002, 942, 249-258.

[32] a) J. Gamby, J.-P. Abid, H. H. Girault, J. Am. Chem. Soc. 2005, 127, 13300-13304; b) J. Gamby, J.-P. Abid, M. Abid, J.-P. Ansermet, H. H. Girault, Anal. Chem. 2006, 78, 5289-5295; c) J. Gamby, M. Lazerges, C. Pernelle, H. Perrot, H. H. Girault, B. Tribollet, Lab Chip 2007, 7, 1607-1609.

[33] J. Turkevich, P. C. Stevenson, J. Hillier, Discuss. Faraday Soc. 1951, 11, 55-75.

[34] H. B. Weiser, Colloid Chemistry, Vol. 2, Wiley, New York 1949, p. 440.

[35] a) M. J. Tarlov, E. F. Bowden, J. Am. Chem. Soc. 1991, 113, 1847; b) S. Song, R. A. Clark, E. F. Bowden, M. J. Tarlov, J. Phys. Chem. 1993, 97, 6564; c) J. D. H. Glenn, E. F. Bowden, Chem. Lett. 1996, 5, 399-400.

[36] C. E. Jordan, B. L. Frey, S. Kornguth, R. M. Corn, Langmuir 1994, 10, 3642-3648.

[37] F. Boulmedais, M. Bozonnet, P. Schwinte, J.-C. Voegel, P. Schaaf, Langmuir 2003, 19, 9873-9882.

[38] V. S. Murthy, J. N. Cha, G. D. Stucky, M. S. Wong, J. Am. Chem. Soc. 2004, 126, 5292-5299.

[39] M. Stastna, K. Slais, Electrophoresis 2005, 26, 3586-3591.

[40] M. A. Roberts, J. S. Rossier, P. Bercier, H. H. Girault, Anal. Chem. 1997, 69, 2035-2042.

Received: August 29, 2007

Revised: February 4, 2008

Published online: April 17, 2008 